\title{
Protée
}

\section{Crénelage, capiton et métadiscours (où l'image numérique résiste à la ressemblance)}

\section{Anne Beyaert-Geslin}

Volume 32, numéro 2, automne 2004

L’archivage numérique : conditions, enjeux, effets

URI : https://id.erudit.org/iderudit/011175ar

DOI : https://doi.org/10.7202/011175ar

Aller au sommaire du numéro

Éditeur(s)

Département des arts et lettres - Université du Québec à Chicoutimi

ISSN

0300-3523 (imprimé)

1708-2307 (numérique)

Découvrir la revue

Citer cet article

Beyaert-Geslin, A. (2004). Crénelage, capiton et métadiscours (où l'image numérique résiste à la ressemblance). Protée, 32(2), 75-83.

https://doi.org/10.7202/011175ar
Résumé de l'article

L'auteure observe différentes utilisations du crénelage dans l'image. Loin d'être une défaillance technique, celui-ci témoigne en effet d'une activité métadiscursive signalant la modernité de l'image. Utilisé comme procédé de masquage de l'identité, le crénelage témoigne d'une crise modale complexe. 


\section{CRÉNELAGE, CAPITON ET MÉTADISCOURS (OÙ L'IMAGE NUMÉRIQUE RÉSISTE À LA RESSEMBLANCE)}

ANNE BEYAERT-GESLIN

Le crénelage est cet artefact qui traduit, par une forme d'escalier, la perte de continuité d'un signal. Cette conversion de l'arrondi et de la diagonale en angle, inhérente aux images à faible définition, peut être compensée par une opération intitulée, selon le cas, anticrénelage ou lissage. L'effet d'escalier est alors supprimé ou, à tout le moins, atténué par un calcul des moyennes de tracés ${ }^{1}$, restaurant ainsi courbes et obliques. Aléa technique susceptible d'être surmonté par des technologies aux performances tout à fait moyennes, le crénelage caractérise pourtant de nombreuses signalétiques et images actuelles où il témoigne, hors de toute défaillance technique, d'une stratégie discursive de l'énonciateur. Le crénelage assume en ce cas une fonction métadiscursive et clame l'origine numérique du texte ou de l'image. En reconduisant le texte ou l'image à son «langage propre», cette insistance suffit à lui accorder une visée moderniste conforme aux conceptions de C. Greenberg (1966: 102)².

Je souhaiterais observer l'activité métadiscursive de quelques hyperboles numériques, telles qu'elles apparaissent en typographie, dans l'image numérique, mais aussi, et avec une préséance historique, dans l'image graphique. Puis, je m'intéresserai aux capitons numériques des images et montrerai comment l'activité métadiscursive circonscrit une crise modale et ajoute une épaisseur narrative. Ces divers exemples convergent solidairement vers le modèle de la mosaïque instruit notamment par L. Dällenbach $(2001)^{3}$; cependant, ils témoignent d'une attention toute sémiotique qui s'attache à percer l'intimité de cette forme globale pour en préciser le fonctionnement, la signification, et fournir l'esquisse d'une première typologie. Le parcours tend ainsi à montrer comment, rompant avec la ressemblance et cessant de décrire le monde, l'image s'interroge elle-même.

\section{CRÉNELAGE ET SIGNALÉTIQUE}

Si le crénelage convertit la courbe en angle, c'est parce qu'il se fonde sur la géométrie élémentaire du pixel (contraction de "picture element», point image). Plus petite partie homogène d'une image numérique, le pixel sert d'unité de mesure pour apprécier sa définition. Pour une même image, son nombre diffère en effet selon le support; lors de l'affichage, il dépend de l'espacement propre à l'écran 
et lors de l'impression, de la qualité de l'imprimante et du grain du papier ${ }^{4}$. Les dimensions de cette unité d'affichage sont d'environ un demi-millimètre sur un demi-millimètre ${ }^{5}$ et les trois luminophores (ou phosphors), émetteurs de lumières rouge, verte et bleue, disposés côte à côte dans le sens horizontal, composent approximativement un carré 6 . Cette forme géométrique imprime tout l'imaginaire du numérique, telle une métaphore incontournable qui, en un inlassable mouvement, associe dans la mémoire du discours le carré génératif du pixel et le carré englobant de l'écran. Une telle insistance de l'angle fût-il d'un rectangle ou d'un carré - a été notamment soulignée par l'artiste informaticien John Maeda:

Le moniteur que nous utilisons est rectangulaire, les pixels qui le remplissent le sont également, les fenêtres qui recouvrent notre interface, ses boutons, ses curseurs et ses commandes, tout est rectangulaire. L'écriture de tout programme (informatique) repose sur l'hypothèse fondamentale de la rectangulaire. (Maeda, 2000: 9)

L'accentuation de la forme carrée a été popularisée par la signalétique, qui produit ainsi un effet de sens «contemporain». Elle s'incarne en d'innombrables typographies actuelles, celle de l'école Camondo ou, plus marquée, celle du Palais de Tokyo qui, de la typographie des toilettes jusqu'au sous-titrage des films, marque ainsi son inscription résolue dans le temps présent. À la suite d'A.-M. Christin (1998: 5), et au vu de son ample diffusion, on pourrait se demander si le caractère crénelé ne condense pas «l'ensemble (du) mode de pensée» de notre époque, à l'instar d'innovations typographiques antérieures, par exemple le didot, inventé au XIXe siècle.

Ces effets de crénelage nous sont en tout cas déjà familiers. Le sens qui leur est attaché trouve diverses déclinaisons et soutient la plupart du temps un mouvement dialectique fort intéressant. Dans un texte consacré aux logos et aux pictogrammes, J. Fontanille (2002: 296) observe, par exemple, l'ambivalence du logo de la région Limousin, la feuille de châtaigner régionale présentant un contour curviligne d'un côté et, suivant l'inspiration numérique, une dentelure anguleuse de l'autre. Une telle conversion de la courbure en angle introduit alors une connotation de "technique avancée», précise l'auteur, connotation qui entre en résonance, à moins qu'elle ne contredise la proposition résolument naturelle de la feuille de châtaigner. Le système semi-symbolique ainsi établi recoupant l'opposition canonique nature/culture, l'identité se construit donc sur un discours mythique. Cette ambivalence caractéristique de la structure économique du Limousin et de ses aspirations identitaires imprime la signalétique de plusieurs institutions, révélant implicitement, et en dépit du tiraillement, une certaine homogénéité régionale. Elle a été reprise notamment par le musée départemental de Rochechouart, écartelé entre deux univers de référence: une vocation historique, d'un côté (il investit un château de la Renaissance parfaitement restauré), et sa mission de présentation de l'art contemporain, de l'autre. Dans cette signalétique, le télescopage des deux époques aboutit au montage attendu du profil du château et d'une typographie crénelée.

\section{CRÉNELAGE ET IMAGE}

Fréquemment exploités dans la signalétique publicitaire, les crénelages sont aussi, plus largement, des figures familières des images. Avant d'envisager divers usages possibles, il convient de distinguer le pixel numérique du pixel graphique.

Un point commun essentiel réunit tout d'abord les deux types de pixel: ils élaborent l'image sans recours au geste (le geste énonciatif du peintre), à partir d'une forme programmatique et sur un principe itératif?. Ainsi constituent-ils une structure à la fois discrète (un agrandissement de l'image révèle les "points») et continue (l'impression juxtapose les "points»). En dépit de ces ressemblances "génétiques", pixel graphique et pixel numérique procèdent néanmoins de technologies et de formes schématiques distinctes.

En effet, si le pixel numérique est angulaire, son précurseur, le pixel graphique, témoigne, sous forme de points ronds, de la juxtaposition des couleurs de base de la quadrichromie. Ces points de la photogravure 
(ou «dots») sont une caractéristique du pop art, des œuvres de Roy Lichtenstein tout particulièrement. Ils «trament» aussi les œuvres d'Alain Jacquet et du mec $a r t^{8}$ et, à une époque plus récente, les peintures de Sigmar Polke, par exemple. Chaque fois, le pixel graphique manifeste une fonction métadiscursive et produit un discours réflexif par lequel, affirmant leur parenté, les œuvres se revendiquent de l'impression mécanique.

Loin d'être un épiphénomène, un tel discours sur l'origine est une façon commune, pour Greenberg, de s'inscrire dans la modernité. Comme j'ai tâché de le montrer par ailleurs, il s'impose comme une constante de l'art depuis la fin du XIX ${ }^{e}$ siècle et procède par une accentuation différenciée de la texture, de la lumière ou de la couleur, chacune de ces dimensions constituant un nouvel «ajout à la ressemblance» ${ }^{9}$. En guise de premier exemple, nous pourrions évoquer l'accentuation de la lumière dont témoigne, de la façon la plus littérale, le pointillisme, lequel, par l'exclusion des mélanges et la juxtaposition des touches, produit un mélange optique qui conserve toute la luminosité des couleurs. Plus largement, l'impressionnisme procède sur ce principe, la référence à ce mouvement permettant d'ailleurs de mettre à jour un système de compensation tensive. En effet, à suivre ces différentes variations intentionnelles, on s'aperçoit que l'accentuation d'une composante du visible entraîne nécessairement l'atténuation d'une autre, de la même façon que l'éclat s'oppose au diffus et qu'un terme intense convoque un terme extense. Pour l'impressionnisme, l'asymétrie se conçoit comme une accentuation de la lumière et une atténuation concomitante de la texture. Avec d'autres mots, J. Ninio a observé un tel déplacement et déploré la perte du rendu textural:

Le souci de traiter de manière homogène les objets les plus divers: arbres, personnages, maisons, a conduit les peintres impressionnistes et leurs successeurs à gommer toute distinction texturale à l'intérieur d'un tableau. ${ }^{10}$

Une autre option consiste, au contraire, à valoriser la dominante texturale. C'est le cas, par exemple, de
Carré blanc sur fond blanc de Malevitch (1918), tableau qui procède par atténuation de la dominante éidétique (Malevitch assure d'ailleurs s'être transfiguré dans le «zéro des formes» ${ }^{11}$ ) au profit de la texture, le contraste de granulosité assumant alors la distinction de la figure blanche sur le fond blanc ${ }^{12}$. Nous pourrions multiplier les exemples et citer d'autres variations intentionnelles par lesquelles l'art du XXe siècle oppose l'éidétique à la texture, à la couleur ou à la lumière, la seule exception revendiquée à ces formulations adversatives, l'hyperréalisme, prêtant elle-même à discussion ${ }^{13}$. Pour notre démonstration, il suffira de voir dans ces accentuations, ces ajouts à la ressemblance, une activité métadiscursive, c'est-à-dire réflexive et commentative, par laquelle l'œuvre signale toujours son origine: la peinture se revendique comme peinture et la sculpture, en exagérant l'amplitude d'un mouvement ou en ajoutant des côtes au thorax du modèle, se signale toujours comme sculpture.

\section{LE PIXEL GRAPHIQUE}

Caractéristique des mouvements modernistes, cette activité métadiscursive se retrouve donc dans les textures à base de pixel graphique, où l'hyperbole texturale permet de se revendiquer de la bande dessinée. C'est le cas dans l'œuvre de Roy Lichtenstein, notamment dans White Brushstroke (1965), qui développe, en outre, un mouvement dialectique très intéressant. Dans ce tableau, la texture à base de points de quadrichromie maintient l'ancrage dans l'univers graphique, tandis que la dimension éidétique renvoie aux beaux-arts par la figure d'une touche de brosse. Témoignant d'une remarquable prise de distance épistémique, l'œuvre concilie même deux conceptions de la texture: la texture graphique, ayant statut de fond, assume une fonction indicielle vis-à-vis de la texture picturale ayant statut de figure. Pour le dire autrement, la texture graphique conserve sa fonction d'ostension, tandis que la texture picturale devient une représentation.

Si elle apparaît de façon tout à fait ponctuelle dans le tableau de Lichtenstein, une telle dialectique, féconde, quoique chargée de tensions schizophrènes, 
est une constante de l'œuvre de l'artiste allemand Sigmar Polke. En dépit de ressemblances superficielles avec l'œuvre de Lichtenstein, l'effort ne consiste pas, en ce cas, à opposer l'éidétique et la texture, la figure et le fond, la visée mimétique et ses moyens, mais il tend plutôt à enfreindre le principe de régularité du pixel graphique, à miner la continuité texturale par le discontinu. La rivalité entre les deux procédures énonciatives se laisse observer, par exemple, dans des peintures Vase II (1965) et Sans titre (1994), où la rotondité approximative et l'irrégularité des pixels, s'ajoutant à une constellation de touches discrètes, trahissent la participation de la main du peintre.

Toutes ces ruptures, dans la continuité de l'impression, permettent aux pixels de Sigmar Polke d'incarner deux notations conventionnelles différentes, fondées l'une comme l'autre sur un contraste de tonalité. Tantôt, quand la trame reste régulière, le pixel exprime un effet de texture (la texture particulière du papier peint, par exemple, dans Vase II); tantôt, lorsque la trame devient irrégulière, il traduit plutôt un effet de modelé (le volume du vase). Si, en renvoyant ainsi à la texture ou au modelé, ces systèmes symboliques distincts montrent comment l'artiste se réapproprie les procédés de la représentation, ils témoignent surtout d'une complexification de l'activité métadiscursive. Ici, le discours sur l'origine est de nouveau ambivalent, mais c'est la texture qui devient en quelque sorte schizophrène puisqu'elle revendique le caractère mécanique de la photogravure et qu'elle interrompt l'implacable continuité graphique, le geste du peintre.

En combinant ces deux praxéologies de la touche, la trame graphique versus le geste du peintre, termes contraires d'une même catégorie, la logique narrative de Polke assume donc, elle aussi, un discours mythique sur l'art. Surtout, elle interroge le fondement épistémologique de l'œuvre, le rapport à l'énonciation pour éprouver les notions d'art allographique et autographique, telles que les ont instruites Goodman et Genette: le pixel graphique, autorisant la multiplication des exemplaires valides de l'œuvre, renvoie à une conception allographique, tandis que le geste du peintre assume un ancrage autographique ${ }^{14}$. Polke résout cependant ce conflit de l'allographique et de l'autographique de façon très particulière. Tandis que Lichtenstein manifestait la tension entre deux possibles de l'énonciation, l'actant individuel qu'est l'artiste et l'impénétrable instance générique que dissimule l'impression graphique, Polke livre l'un à l'autre. La touche particularisante du peintre interrompant en fin de compte la logique généralisante du pixel graphique - l'artiste «remporte» l'énoncé.

\section{LE PIXEL NUMÉRIQUE}

Sur un présupposé d'angularité et non plus de rondeur, certaines images numériques procèdent à de telles accentuations texturales et manifestent une activité métadiscursive comparable. C'est le cas des publicités de John Maeda ${ }^{15}$, notamment, où l'usage hyperbolique du pixel rappelle le mode d'élaboration de l'image numérique par itération d'unités discrètes. Les images de Maeda sont le résultat d'une méthode invariable. Dans un premier temps, l'artiste choisit une unité programmatique qu'il duplique ensuite jusqu'à la constitution d'une icône globale.

L'unité adoptée est, selon le cas, un carré (métaphore du pixel) ou un objet du monde naturel miniaturisé, par exemple un capuchon de crayonfeutre Too ou un appareil de la gamme Sony ${ }^{16}$. De même que l'unité programmatique, le modèle génératif de Maeda s'apparente à un «objet trouvé» (pour ne pas dire un «ready made»), trouvé dans la nature généralement, puisqu'il reprend le mode d'enroulement du volubilis ou le principe du nombre d'or pour assembler les petites fleurs du tournesol sur le plateau de son capitule. Il faudrait sans doute s'attarder à ces modèles publicitaires qui, lorsqu'ils portent alternativement l'attention sur la partie et sur le tout, thématisent un objet ou l'autre selon le changement de focale: le minuscule ordinateur qui sert d'unité programmatique ou le volcan (Sony) qu'il compose lorsqu'on le multiplie, par exemple. Pour notre étude, il suffira d'observer comment l'accentuation de la texture numérique, dans l'affiche représentant la Joconde réalisée avec une mosaïque de 
crayons-feutres Too, argumente une valorisation utopique de l'objet à vendre en prônant des valeurs existentielles.

Plus précisément, la valorisation est double dans cette publicité. D'une part, il s'agit de conjoindre l'usager à l'art représenté par son chef-d'œuvre ou, au moins, à l'œuvre la plus célèbre du monde, la Mona Lisa. De l'autre, l'accentuation de la texture rappelle le remplissage de l'image par les pixels, c'est-à-dire la générativité du numérique. Aux valeurs pratiques, critiques ou ludiques, telles que les a décrites J.-M. Floch (1990) dans son carré des valeurs publicitaires, l'affiche de Maeda préfère donc les valeurs existentielles et une orientation utopique. En réconciliant l'utopie contemporaine du numérique à celle, traditionnelle, des beaux-arts, pour en proposer la rénovation, elle argumente à nouveau un discours mythique.

Sur l'exemple des publicités de J. Maeda, on vérifie à nouveau que l'accentuation de la texture assume une fonction métadiscursive par laquelle l'image - fûtelle œuvre d'art ou affiche publicitaire - revendique son origine, un mode d'élaboration particulier.

\section{LE CAPITON NUMÉRIQUE}

Nous avons distingué les pixels sur un critère ontologique et schématique (le pixel graphique et rond versus le pixel numérique et carré) et, sur cette base, observé diverses formes d'accentuations métadiscursives. Il convient maintenant de dégager un second critère pour distinguer l'accentuation globale, telle que nous l'avons instruite précédemment, et l'accentuation locale. Sur ce principe, on appellera très librement capiton numérique, sans référence au point de capiton de Lacan, cette zone critique de l'image où le pixel est discrétisé, épaissi, pour produire une effet de crénelage qui fait écart dans la fine granulosité.

Notre premier exemple de capiton apparaît dans le recouvrement d'une photographie de la villa Noailles de Robert Mallet-Stevens par les carrés des pixels 17 . «Recouverte» de gros pixels, la maison - forme pourtant familière - est fragilisée et transformée en hypoicône. Le capiton reste cependant précisément circonscrit au bâtiment, désigné comme zone critique de l'image, et préserve en un grain fin ses alentours: l'arbre à l'arrière, le ciel et la pelouse. Surtout, dans la partie gauche, il détoure avec minutie la silhouette de deux actants qui donnent l'échelle à l'image et ajoutent crédit à l'hypothèse d'une icône de maison. Ainsi retouchée, la photographie croise deux modes d'élaboration, celui du bâtiment de Mallet-Stevens, construit sur la géométrie élémentaire du cube, et celui de l'image numérique. Elle compose ainsi un discours mythique où le premier modèle génératif présentifie le second en accusant, voire en exagérant son orthogonalité, en actualisant en tout cas une géométrie potentielle.

\section{LE CRÉNELAGE PUDIQUE}

Si le crénelage peut rénover l'esthétique fonctionnaliste de Mallet-Stevens, cette application reste somme toute particulière. Plus communément, l'usage médiatique l'associe à un effet de masquage des visages des prévenus dont la culpabilité n'a pas été démontrée, des mineurs ou des témoins qui souhaitent déposer dans l'anonymat ${ }^{18}$. En ce cas, le capiton pudique perturbe l'identification de l'actant en introduisant une zone contrastive au niveau des yeux ou du visage tout entier. Celui-ci modalise alors l'image par un / ne pas pouvoir faire/ (ne pas pouvoir montrer) qui l"inscrit dans la filiation d'autres procédés visuels, tels que le bandeau noir ou l'effet de flou. Ces divers procédés témoignent d'une crise modale, d'une rupture dans le contrat implicite de l'exposition. À l'instar de ces procédés antérieurs, le capiton numérique se laisse identifier à une marque légale, déontologique ou morale, une petite zone critique où le caché s'incruste dans le montré afin de garder secrète l'identité de l'actant.

S'il s'inscrit dans cette filiation, le capiton manifeste toutefois une compétence modale plus complexe. En effet, il procède en dissolvant les traits du visage sous des pixels démesurés, supprimant dès l'abord toute possibilité d'intersubjectivité et laissant à l'observateur le loisir de "positionner", à partir de différences chromatiques et tonales, des yeux et une 
bouche susceptibles de restaurer l'empathie. Or, si l'image apparaît toujours stabilisée à l'écran ou dans le magazine, on s'aperçoit qu'un changement de focale ou une modification du format des unités constitutives du capiton - opération élémentaire de l'informatique - suffisent à réduire le contraste et à «lisser» le visage. Le capiton est donc une figure extrêmement instable, résultat du fragile ajustement de la dimension du pixel à une distance d'observation: la perception peut donc tout aussi bien le contrarier et «lisser» l'image.

Une telle instabilité n'est pas sans conséquence pour le contrat modal du capiton, qui ne garantit guère l'anonymat de l'actant ou, plutôt, qui ne le préserve que sous certaines conditions de stabilisation du pixel (une équation format/distance) et dans une lecture immédiate. En somme, le capiton pudique n'est jamais qu'une promesse de protection, assumée selon certaines conditions de la perception. Mais on pourrait aller plus loin et se demander si, indiquant le visage qu'il masque si mal, le capiton ne s'attache pas, à défaut d'un / pouvoir faire/ (pouvoir masquer), des compétences de /vouloir faire/ et de /devoir faire/ (vouloir cacher, devoir cacher). Entre /devoir faire/ (devoir cacher) et/ne pas pouvoir faire/ (ne pas pouvoir cacher), s'insinuerait donc une sémiotique non plus déontologique, mais déontique, qui établirait sa duplicité. Non seulement le capiton ne masque guère l'identité de l'actant, mais, par la valorisation déontique, il l'indique même comme un actant à protéger et l'expose comme victime. Ainsi constitue-t-il un objet de savoir qu'il ajoute à l'épaisseur narrative de l'image, un objet véridictoire, c'est-à-dire un secret qui pourra attiser la curiosité de l'observateur ${ }^{19}$.

Le capiton pudique peut dissimuler un visage, mais également d'autres parties de l'anatomie. Son usage nous est alors si familier qu'on ne s'étonne pas de le voir garantir la pudeur des Sims, nos petits alter ego mondialisés sur cédéroms ${ }^{20}$, lorsqu'ils se rendent dans la salle de bains. En retouchant localement ces petits personnages de fiction ou en recouvrant intégralement leur corps nu, le crénelage pudique manifeste alors une activité métadiscursive spécifique, où «l'effet de secret» se trouve particularisé et augmenté d'un «effet de vie». Puisque cette petite figure mouvante doit être ainsi dissimulée à nos regards indiscrets lorsqu'elle se rend aux toilettes, c'est qu'elle n'est pas un simple artefact technologique, mais s'apparente bien à un être humain dont il faut, comme il est de mise dans les médias, préserver l'intimité. En ce sens, le capiton, qui dissimule sa nudité, témoigne d'une actantialisation, sinon de cet «effet de réalité» que Barthes accordait déjà au détail ${ }^{21}$. Indice d'une zone intime du corps, le crénelage pudique devient alors un symbole du principe actantiel.

\section{NUMÉRIQUE ET CELLULITE}

La publicité télévisée exploite le capiton numérique comme support métaphorique d'une promesse de réduction du capiton de cellulite (encore appelé peau d'orange). Puisque de telles images dévoilent largement le corps féminin, on pourrait s'attendre à ce que le capiton numérique vienne signaler un irreprésentable de l'image, un "ne pas pouvoir montrer». Or la zone que les capitons montrent/cachent n'est pourtant jamais une zone impudique de l'anatomie, mais une petite localité particulière située en haut de la cuisse. Un tel déplacement tend à accréditer l'hypothèse selon laquelle le crénelage ne traduit pas, en ce cas, un effet de pudeur, mais plutôt l'accomplissement d'un programme narratif: il indique un dysfonctionnement localisé de l'épiderme et promet une transformation.

Les produits de soin corporel disposent de nombreuses métaphores du grain de la peau qu'ils déclinent en fonction du principe actif recherché: l'hydratation (un fond de désert aride), le lissage (un papier froissé), le raffermissement ou l'amincissement, etc. Dans la même logique rhétorique,

l'épaississement du pixel semble être une métaphore judicieuse, susceptible de décrire le phénomène de la cellulite comme un petit amas graisseux local. Ainsi, la publicité impose-t-elle la rassurante homologation de l'épaississement numérique et de l'épaississement cutané, du grain fin de l'image et du grain fin de 
l'épiderme. Pourtant, le capiton numérique s'offre comme une métaphore bien plus féconde qui argumente, au-delà de la simple ressemblance ${ }^{22}$, une véritable dramaturgie, où l'épaississement (pixel épais) précède la réduction (pixel fin) et pose la fine granulosité du corps mince comme visée du programme aspectuel. Le capiton corporel peut se résorber aussi aisément qu'un capiton numérique se transforme, par la banale et mécanique opération du lissage, en une image de grande finesse, nous assure-ton.

Il reste, pour établir la pertinence de la métaphore, à poser le grain fin comme l'aboutissement obligé du programme aspectuel. On s'aperçoit en effet que la métaphore repose essentiellement sur l'horizon d'attente particulier du numérique qui suppose un rendu extrêmement précis, un "piqué » ${ }^{23}$. Cette finalité prescrit un déroulement aspectuel intangible où l'épais, faisant écart, précède nécessairement le fin, norme véritable de l'image numérique. La figure cidessous montre la symétrie des deux transformations, par la voie du lissage numérique et celle de la crème lissante.

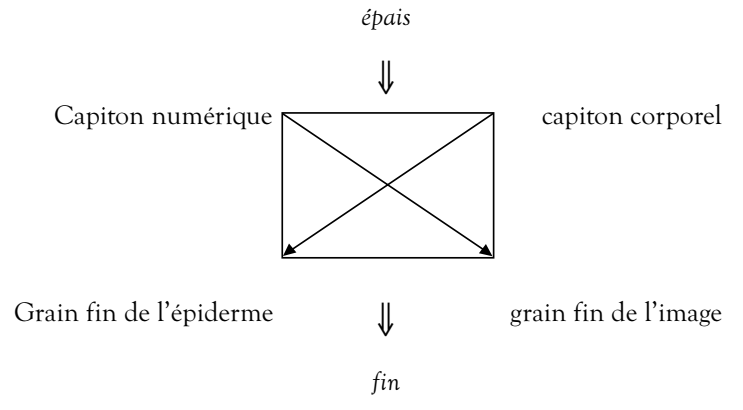

\section{CONCLUSION}

Si ce bref parcours ne rend pas compte de façon exhaustive de l'activité métadiscursive des hyperboles numériques, telles qu'elles apparaissent dans des genres très divers, du moins livre-t-il quelques indications sur une certaine façon de penser la «modernité»:

- Le pixel numérique s'impose tout d'abord comme une métaphore visuelle extrêmement féconde.
Profitant de la forme du carré, il peut en effet actualiser et rénover des systèmes de représentation antérieurs construits sur une géométrie élémentaire: de l'archaïque mosaïque au suprématisme de Malevitch ${ }^{24} \mathrm{ou}$, en architecture, au fonctionnalisme, par exemple. De plus, en autorisant la collusion de deux systèmes de représentation, voire leur mise en abyme, il peut soutenir un discours mythique où l'image pense son origine à l'aune du modèle numérique. Sa fécondité métaphorique tient encore au fait qu'en posant la finesse comme une visée obligée du numérique, la norme contre laquelle l'épaississement fait écart, il suggère un processus aspectuel de réparation et une sorte de dramaturgie latente. - Ensuite, loin d'être des épiphénomènes, ces accentuations de la texture s'inscrivent dans une continuité moderniste qui tend toujours à catégoriser les dimensions du visible et qui s'affranchit de la visée mimétique pour commenter son propre modèle génératif. Pour prendre toute la mesure de l'épaisseur sémantique qu'elles ajoutent au discours, il faut donc les concevoir comme des points de résistance à la ressemblance promise par l'extrême précision du médium. Par ces points, l'image interrompt elle-même la visée mimétique et renonce à cette connivence avec le monde naturel de la photographie que Barthes décrivait déjà comme un «ça a été» (1994: 1165 notamment).

- Lorsque ces hyperboles posent leurs zones critiques, c'est donc très logiquement pour cesser de décrire le monde, pour faire retour sur l'image et expliciter son modèle génératif. Ce commentaire réflexif se conçoit également comme une façon de sensibiliser l'image, d'indiquer où le bât (son statut épistémique) blesse et demande précaution. En ce sens, si la multiplicité des références au modèle génératif numérique porte témoignage d'une vaste adhésion, voire d'une euphorie numérique, il resterait à tempérer cet effet de sens trop général en ajoutant qu'elles sont avant tout une façon de s'inquiéter de notre relation aux images. 


\section{NOTES}

1. Deux dictionnaires, emblématiques de cultures éléctroniques légèrement différentes, m’ont éclairé sur ces notions techniques: celui de J. Notaise et alii (1995) et celui de L. Poissant et alii (1997). Le premier décrit les opérations symétriques crénelage/lissage aux pages 275 et 511; celui de Poissant conçoit le lissage comme une opération de simulation de la continuité d'éclairement dans le rendu d'une surface courbe.

2. Cette thèse rejoint les conceptions de Cézanne qui voulait déjà «être peintre avec les qualités mêmes de la peinture», cité par É. Bernard (1978: 51). Le texte que Greenberg a consacré à l'art de Cézanne confirme la modernité du peintre de la Sainte-Victoire (1989: 59-68).

3. Pour Dällenbach, la mosaïque pourrait relever d'un mood mosaïque dont tous les esprits «subiraient l'empire». Succédant au puzzle des années 1970 et 1980, elle serait le modèle culturel marquant depuis les années 1990, comme cet auteur le suggère (p. 18). Je remercie J. Baetens de m'avoir indiqué cette référence.

4. Parmi les nombreuses définitions du pixel, ma préférence va à la plus consensuelle, dans Notaise et alii (1995: 657).

5. Les dimensions sont exactement de 0,5 sur $0,45 \mathrm{~mm}$. Ses caractéristiques sont décrites plus précisément dans B. Caillaud (2001: 49sq.)

6. «Le "vrai” pixel moyen, celui que l'on peut trouver sur un affichage cathodique, n'est jamais parfaitement carré, il est plus flou qu'autre chose", estime J. Maeda. Pour cet auteur, le pixel est constitué de "petits éléments rayonnants rouges, verts et bleus de forme oblongue " (Maeda, 2000: 64-65 et 66-67).

7. J'ai tâché de mettre en évidence ce principe itératif (et non récursif), tel qu'il est érigé en esthétique (A. Beyaert, 2003a).

8. Si le pop art voit converger deux mouvements, américain et britannique, autour de 1965, son héritier direct, le mec art (peinture mécanique ou art mécanique), se constitue à Paris autour de Jacquet, Rotella, Bertini et Bury et surtout autour du critique P. Restany. Pour préciser la filiation, on se reportera à G. Durozoi (1992).

9. La proposition est argumentée plus patiemment dans A. Beyaert (2003b: 81-90). Le texte considère l'hyperbole texturale comme l'accentuation majeure faite par l'art du XXe siècle, bien avant celle de la lumière et de la couleur.

10. J. Ninio (1996: 63). «Un savoir, qui fait défaut à la science naturelle, s'est éteint", poursuit le biologiste, marquant ainsi une certaine nostalgie des compétences texturales des primitifs flamands. $\mathrm{Si}$ l'on peut comprendre ses regrets, la désaffection doit néanmoins être relativisée. Le manque d'intérêt pour la représentation des textures des peintres du XXe siècle n'est qu'une conséquence prévisible de la mise en cause de la peinture figurative. En revanche, l'accentuation de la texture semble bien être un des «ajouts» majeurs à la ressemblance, et la dimension la plus souvent accentuée par l'art du XXe siècle. L'opposition, entre l'habileté des peintres du XVe siècle à représenter les textures du monde naturel et l'accentuation texturale au XXe siècle, traduit plus fondamentalement deux conceptions différentes de la mimésis, la seconde étant développée notamment par Ricœur. Évoquant son admiration pour l'art de son siècle, le philosophe explique: «c'est lorsque la peinture a cessé, au XXe siècle, d'être figurative, que l'on a pu prendre enfin la pleine mesure de cette mimésis qui a justement pour fonction, non pas de nous aider à reconnaître des objets, mais à découvrir des dimensions de l'expérience qui n'existaient pas avant l'œuvre" (P. Ricœur, 1995: 260).

11. «Je me suis transfiguré dans le zéro des formes et je suis allé, au-delà du zéro, vers la création, c'est-à-dire vers le Suprématisme, vers le nouveau réalisme pictural, vers la création non figurative ». Cette phrase, datée de 1916, était mise en exergue lors de l'exposition Malevitch, un choix dans les collections du Stedelijk Museum d'Amsterdam, présentée au Musée d'art moderne de la ville de Paris (30 janvier au 27 avril 2003).

12. Un regard plus attentif révélerait toutefois que le contraste de texture entraîne nécessairement un contraste chromatique et lumineux, la lumière réfléchissant différemment sur des textures dissemblables. La valorisation d'une composante du visible occasionne donc des accentuations secondaires.

13. Courant apparu dans les années 1965-1970 aux États-Unis, l'hyperréalisme semble en effet renouer avec l'habileté technique des peintres de nature morte. Pourtant il ne se départit jamais d'une distance critique vis-à-vis du monde naturel. Outre sa prédilection pour les couleurs saturées et les surfaces réfléchissantes, il adopte le point de vue de la photographie, endosse sa tradition de reportage et ses motifs. Ainsi met-il en évidence les différences entre la perception naturelle, la représentation de la tradition picturale et celle de la photographie.

14. «Dans certains cas, explique Goodman, la notion d'authenticité a un sens et elle est définie par l'histoire de production d'une œuvre et dans d'autres, elle n'en a aucun et toutes les copies correctes constituent autant d'exemplaires valides de l'œuvre» (dans G. Genette, 1994: 22-23)

15. Une analyse plus complète du procédé de Maeda est proposée dans A. Beyaert (2003a).

16. Le choix de cette unité est une prémisse essentielle car, comme 
l'explique le graphiste, « une forme programmatique correctement construite est par définition de nature variable, mais si l'idée visuelle centrale est médiocre, ses variations le seront également" (J. Maeda, $2000: 250$ ).

17. La photographie est insérée dans la revue Officiel, n²75, mai 2003, p. 172-173.

18. Dans son dictionnaire, L. Poissant identifie cette zone épaissie de l'image à une mosaïque, appellation qui semble être passée dans le langage courant au point de se prêter au néologisme. Dällenbach relève par exemple l'emploi du verbe mosaïquer dans le journal Le Monde du 8 juin 1999 («Sur TF1, tous les visages étaient mosaïqués»). L'activité désignée par ce néologisme serait en fait très ancienne, puisque les iconoclastes ont fréquemment "mosaïqué», par de nouvelles tessères servant de cache, les visages et figures de la mosaïque ainsi interdits à la représentation. La pratique, citée par Dällenbach, est abondamment illustrée par M. Piccirillo (1992).

19. De tels capitons pudiques apparaissent fréquemment dans les talk show. Sans offrir de réelle garantie à l'actant dont ils sont censés protéger l'identité, ils le marquent du sceau d'un secret qui augmente l'intérêt du discours et suscite la curiosité.

20. Les Sims et les disques additionnels (Entre chiens et chats, Surprise Party, etc.) sont des jeux d'Electronic Arts présentés notamment sur [www.thesims.com].

21. L'effet de sens du détail, «supplément à la fois inévitable et gracieux", est commenté dans R. Barthes (1994: 1142 notamment). 22. "Bien faire les métaphores, c'est bien apercevoir les ressemblances" (Aristote, 1980: 1459a 4-8).

23. Cette extrême finesse du numérique a été utilisée dans la publicité pour les appareils photo numérique, lorsque ceux-ci sont arrivés sur le marché du grand public, la joute entre les marques instruisant, jusqu’à l'ivresse, un imaginaire du très grand nombre: deux millions puis trois millions puis cinq millions de pixels, selon l'appareil.

24. Dans ces croisements entre le numérique et les mouvements modernistes, les références au carré sont incessantes. Dans Maeda@ Media, Journal d'un explorateur du numérique, J. Maeda évoque à plusieurs reprises l'œuvre de Malevitch qui, lui-même, célébrait le carré comme un «enfant royal plein de vie».

\section{RÉFÉRENCES BIBLIOGRAPHIQUES}

ARISTOTE [1980]: La Poétique, trad. et dir. par R. Dupont-Roc et J. Lallot, Paris, Seuil.

BARTHES, R. [1994]: La Chambre claire, CEuvres complètes, t. 3, prés. par E. Marty, Paris, Seuil. Bernard, É. [1978]: Conversations avec Cézanne, Paris, Macula. BEYAERT, A. [2003a]: «Esthétique du pixel. L'Accentuation de la texture dans l'œuvre graphique de John Maeda", Communication et langages, $\mathrm{n}^{\circ} 138,23-37$;

_ [2003b]: «Texture, couleur, lumière et autres arrangements de la perception», Protée, vol. 31, n 3 ("Lumières»), hiver 2003-04, 81-90.

CAILlaUd, B. [2001]: La Création numérique visuelle. Aspect du computer art depuis ses origines, Paris, Europia.

Christin, A.-M. [1998]: «Écriture et multimédia », Degrés, n $92-93$

("Penser le multimédia »).

DÄLlENBACH, L. [2001]: Mosaïques, Un objet esthétique à rebondissements, Paris, Seuil.

DuROzOI, G. (dir.) [1992]: Dictionnaire de l'art moderne et contemporain, Paris, Hazan.

FLOCH, J.-M. [1990]: «J'aime, j'aime, j'aime... Publicité automobile et système de valeurs de consommation ", Sémiotique, marketing et communication (sous les signes, les stratégies), Paris, P.U.F., 119-153. FONTANILLE, J. [2002): «Le langage des signes et des images: pictogrammes, idéogrammes, signalétique et publicité. Le pouvoir créateur des signes ", Université de tous les savoirs, vol. 5 (Le cerveau, le langage, le sens), Paris, Odile Jacob.

GenetTE, G. [1994]: L'Euvre d'art. Immanence et transcendance, Paris, Seuil.

GreenberG, C. [1966]: «Modernist Painting», dans G. Baltrock (dir.), The New Art. A Critical Anthology, New York, Dutton;

[(1961) 1989]: «Cézanne», Art et Culture. Essais critiques, trad. fr. de Ann Hindry, Paris, Macula.

MAEDA, J. [2000]: Maeda@ Media. Journal d'un explorateur du numérique, Paris, Thames \& Hudson.

NinIO, J. [(1989) 1996]: L'Empreinte des sens, Perception, mémoire, langage, Paris, Odile Jacob.

NOTAISE, J., J. BARDA et O. DUSANTER [1995]: Dictionnaire du multimédia : audiovisuel, informatique, télécommunications, Paris, AFNOR. PICCIRILlO, M. [1992]: The Mosaïcs of Jordan, Ammân (Jordanie), American Center of Oriental Research.

POISSANT, L. et alii [1997]: Dictionnaire des arts médiatiques, Sillery, Presses de l'Université du Québec.

RICCEUR, P. [1995]: La Critique et la Conviction, entretien avec F. Asouvi et M. de Launay, Paris, Calman-Levy. 